\title{
Strategies of Healthy Adults Walking on a Laterally Oscillating Treadmill
}

\author{
Rachel A. Brady, M.S ${ }^{1}$; Brian T. Peters, Ph.D. ${ }^{1}$; Jacob J. Bloomberg, Ph.D. ${ }^{2}$
}

${ }^{1}$ Wyle’s Life Sciences Group, Houston, TX, USA

${ }^{2}$ Neuroscience Laboratories, NASA Johnson Space Center, Houston, TX, USA

Running Head: Oscillating Treadmill

Abstract Word Count: 142

Main Text Word Count: 2711

Number of References: 30

Number of Tables: 0

Number of Figures: 4

Corresponding Author:

Rachel Brady, M.S.

Wyle Laboratories

1290 Hercules Drive

Houston, TX 77058

Phone: 281-483-6620

Fax: 281-244-5734

Email: rachel.brady-1@nasa.gov 


\section{Abstract:}

We mounted a treadmill on top of a six degree-of-freedom motion base platform to investigate locomotor responses produced by healthy adults introduced to a dynamic walking surface. The experiment examined self-selected strategies employed by participants when exposed to continuous, sinusoidal lateral motion of the support surface while walking. Torso translation and step width were used to classify responses used to stabilize gait in a novel, dynamic environment. Two response categories emerged. Participants tended to either fix themselves in space (FIS), allowing the treadbelt to move laterally beneath them, or they fixed themselves to the base (FTB), moving laterally as the motion base oscillated. The degree of fixation in both extremes varied across participants. This finding suggests that normal adults have innate and varied preferences for reacquiring gait stability, some depending more heavily on vision (FIS group) and others on proprioception (FTB group).

Keywords: Human locomotion, Unstable surface, Treadmill, Adaptation, Stability

\section{Introduction}

Human ability to adjust gait patterns has been explored with manipulations of optic flow, body loading, and the support surface, and each modality has been successful in inducing adaptive locomotor changes [1-9]. Subjects who walked on a treadmill while viewing immersive, rotating visual scenes showed adaptive changes both during their exposure and during post-exposure testing [5, 6, 8]. Prokop et al. showed that optic flow modulates a selfselected walking velocity and suggested that an individual's strategy to stabilize walking velocity depends on the way information is weighted from different afferent sources [7]. 
The role that support surface plays on gait patterning has been addressed as it relates to obstacle avoidance [3, 4], perturbations [9], and locomotor aftereffects [1], but locomotor responses to a continuously oscillating support surface have not been explored.

Warren et al. [10] examined the directional specificity of postural sway responses in healthy adults who walked on a treadmill while viewing a moving hallway scene. At the outset of the study, it was known that only one-half to two-thirds of participants exposed to visual stimulation alone demonstrated a significant sway response when standing [11]. Anticipating similar behavior from walking subjects, Warren and co-workers set a criterion that non-responders would be dropped from the analysis. Four of nine participants were dropped because they did not demonstrate a significant sway response to the hallway scenes, and the team went on to investigate what set these individuals apart from those who did respond. One explanation was that the sinusoidal nature of the displays used in the experiment was too easily anticipated and therefore discounted. Another was that the unaffected subjects did not exhibit significant sway because they were making a conscious effort to avoid doing so. Both explanations were discounted experimentally, leading the team to conclude that "it is possible that there are individual differences in the relative reliance on visual and somatosensory information under conflict conditions, which could account for the failure of some observers to respond to purely visual oscillations”.

This study presents the inverse scenario—support surface variation with a stationary visual scene. The goal of this research was to further characterize response strategies in healthy adults during conflict conditions, specifically during treadmill walking on an oscillating surface. 
2. Methodology

\subsection{Equipment}

Support surface variation was produced by a commercially available, six degree-of-freedom (6 DoF) electric motion base (Moog, East Aurora, New York) on which a standard treadmill (Quinton Model Q55, A-H-Robins Company, Philadelphia, PA) was mounted. In-house software written in Matlab (Mathworks, Natick, MA) was used to program a $25.4 \mathrm{~cm}$ peak-topeak lateral, sinusoidal translation of the motion base and the treadmill's belt speed was set at $1.12 \mathrm{~m} / \mathrm{s}$ (2.5 mph). A computer-generated, static outdoor scene was back-projected onto a 3.6 m x $2.6 \mathrm{~m}$ screen positioned $2 \mathrm{~m}$ in front of participants. Because the treadmill surface was significantly elevated above the surface of the floor, the visual scene was used to raise the visual reference frame to a height coincident with the walking surface of the treadmill.

A separate study aim prompted the division of participants into two groups receiving the lateral base oscillations at either $0.2 \mathrm{~Hz}$ or $0.3 \mathrm{~Hz}$. When data were analyzed, no differences between the groups were present in the variables described here so the groups were combined.

\subsection{Participants}

Nineteen healthy adults (ten men), average ( \pm 1 SD) age $36( \pm 9)$ years, all naïve to the lateral translation motion profile, volunteered for this study. All participants completed a modified Air Force Class III physical prior to testing and provided written informed consent. The study was approved by the Committee for the Protection of Human Subjects at NASA's Johnson Space Center.

Participants were instructed to walk as naturally as possible, which included our preference that they avoid handrail use and not look at their feet. One subject, not included in the count of 
nineteen, was dropped from analysis for handrail use, but all others were able to comply with both conditions. To minimize distractions, operators did not initiate conversation during the test other than to relay the time remaining in the test. Participants, on the other hand, were free to share their comments or impressions at any time.

\subsection{Procedures}

Subjects were secured in a non-restrictive safety-harness and began the data collection with a two-minute period of normal treadmill walking. A five-cycle ramp-up period was used to transition the base from its stationary state to its programmed motion profile. The final motion profile was sustained for twenty minutes.

\subsection{Data Collection and Analysis}

Three-dimensional kinematic data from the torso and feet were collected at $60 \mathrm{~Hz}$ (Motion Analysis; Santa Rosa, CA). Retro-reflective spherical markers (25 mm dia.) affixed to a vest were positioned approximately at C7 and bilaterally at T12. Foot markers of the same size were positioned bilaterally near the soles of the shoes at the fifth metatarsals and beneath the lateral malleoli. Markers were placed on the treadmill to provide points of reference during base motion. Kinematic data for the motion base, torso, and feet were low-pass filtered with a recursive second-order Butterworth at cut-off frequencies of $1 \mathrm{~Hz}, 5 \mathrm{~Hz}$, and $10 \mathrm{~Hz}$, respectively.

Custom Matlab programs were used for data analysis. Data from the final minute of the normal walking period were used to calculate each subject's baseline average step width.

Torso motion was defined using the midpoint between the two T12 markers. The lateral position of the treadbelt midline was subtracted from this point to present the lateral torso position with respect to the treadmill. A Fourier analysis was performed on the resulting waveform for analysis of the signal in the frequency domain. A similar analysis was performed 
on the time series data representing the lateral motion of the treadmill. A ratio between the amplitudes of torso and base motion, at the predominant base motion frequency, was used to investigate the influence of the lateral treadmill motion on the lateral torso position.

The positions of the markers placed beneath the lateral malleoli were used to determine step width. The lateral position of each foot was determined relative to the treadbelt at each foot's respective midstance point. The difference between these locations was defined as the step width. It is important to note that only the step widths measured from the right foot to the left foot are reported here. The left-to-right step widths produced biases in the direction opposite those shown in the Results section below.

\section{Results}

During the data collections, we observed that most participants translated laterally with the base but a few held a fixed position and let the base translate beneath them. The terms 'fixed-tobase' (FTB) and 'fixed-in-space' (FIS) were adopted to discriminate between these groups.

Lateral torso translation characteristics of a representative FTB and FIS walker are illustrated in Figure 1. Figure 1a depicts lateral torso and base positions with respect to space. When the base position is subtracted from the torso position (Figure 1b), a distinct difference emerges between the resulting waveforms. Figure 1c shows the FFT output of the lateral torso position, after subtracting the base position, calculated from the last ten minutes of the base motion period. We restricted the analyses to the latter portion of the data to avoid the initial variability present as subjects adapted to the underfoot motion. (An in-depth investigation of this adaptation period is beyond the scope of this report and will be addressed in a subsequent paper.) The power in the FFT output near $1 \mathrm{~Hz}$ represents normal torso oscillations present in gait. Power spikes at the 
base frequency represent a subject's effort to oppose the lateral oscillation and remain stationary with respect to space.

We used a ratio between the FFT amplitude of the torso and base data, measured at the base frequency, to quantify the effects of the base motion on the torso. Figure 2 shows the torso-tobase ratios for the last ten minutes of each subject's trial. This ratio for most participants falls on a continuum between 0.05 and 0.3, a range that encompasses FTB walkers. A significant step increase in the ratios separates a smaller group of four FIS participants from the rest. The torsoto-base ratio for these four FIS participants is above 0.4 .

Our initial investigation of the step width data was intended to uncover a hypothesized increase in step width to effectively increase the subject's base of support. A comparison of the mean values of the last minute of normal treadmill walking to the first minute of the steady-state portion of base motion does indeed show an increase in step width $(\mathrm{p}=0.002)$. A more striking result from the time-series data was the increase in variability that remained elevated throughout the trial. To account for the effects of the base movement on step width, data were plotted using a polar plot. Examples are shown in Figure 3. The angular coordinate of the polar plot defines the state of the base with the horizontal axis representing the base position and the vertical axis representing the base velocity. The distance from the center of the plot to each data point is the right-to-left step width that has been normalized to the average step-width value prior to the start of base motion. The radius and center of the best-geometric-fit circle are used to quantify the results. A radius above a unity value indicates an increase in average step width. A deviation of the center of the circle indicates that step width is being modified in a systematic way relative to the motion of the base. Each of these effects can be observed in the data in the figure. 
The radii of the circles fit to the final ten minutes of data for all participants indicate that the average step width remained wider than normal even after participants had adapted to the base motion (range: 0.95 to 1.31, mean: $1.08, \mathrm{p}=0.003$ ). Eccentricities of the circle fit are presented in Figure 4 where the centers of the circles are plotted. The two shaded regions on the plot show \pm 1 standard deviation around the mean of the angular position of the data points for the FIS and FTB groups. Note that because the angular position of data points lying near zero are increasingly unreliable, three data values with eccentricities less than 0.05 were not included in these mean calculations. FIS and FTB groups show fundamentally different strategies in their foot placement during base motion. The FIS walkers show a downward shift in their bestgeometric-fit circle, indicating that the left foot steps wider when the base is moving right and narrower when the base is moving to the left. The step-width of the FTB walkers is either not affected significantly by the base motion or has the opposite tendency of the FIS walkers.

\section{Discussion}

Participants who walked on a laterally translating motion base could be classified into two groups: those who remained "fixed to base" and followed the treadbelt side to side as it translated, and those who remained "fixed in space", letting the treadbelt translate beneath them. Similar classifications have been observed in subjects standing on a linear accelerator translating in the A-P direction [12]. We believe this indicates that some individuals maintain stability by relying primarily on visual feedback while others rely primarily on proprioceptive feedback.

Some populations have been shown to be either more visually or proprioceptively dependent. For example, autistic children performing an adaptation task exhibited a dominance of proprioceptive weighting over visual weighting [13] and elderly subjects performing an obstacle avoidance task were more visually dependent than their younger counterparts, perhaps due to an 
impairment of proprioceptive mechanisms [14]. Findings by Keshner et al. [12] suggest that preferences in sensory weighting may exist in the normal population as well. When healthy, standing subjects received fore-aft translations on a support surface while viewing an earth-fixed visual scene, two response strategies emerged. Some participants used an 'inverted pendulum’ strategy, in which the head, trunk, and shank moved in the same direction as the sled. Others used a 'hip strategy', in which the head and trunk moved opposite the direction of the sled but the shank moved in the direction of the sled. It could be the case that one group moved with the sled because they relied more heavily on proprioceptive feedback, whereas the other moved opposite the sled to stabilize the head in space and maintain a visual reference.

In addition to similar fixed-to-base vs. fixed-in-space observations made in this study, other behaviors can be qualitatively generalized. All participants exhibited an initial startle response at motion onset, frequently likening the sensation to a "drunken stagger”, and then gradually settled into a comfortable and repeatable walking pattern, generally within five minutes of the twenty minute trial. Some participants commented about a peripheral awareness of the brightly colored emergency shutoff switch mounted on the handrail in front of them. The degree to which this awareness influenced their walking strategy is not known, so future studies will incorporate field-restriction goggles to remove this base-fixed visual reference.

Interestingly, average step width plots for most FIS walkers were relatively flatlined when plotted on a traditional x-y axes. A FIS walker spends half the base's cycle taking a narrower right-to-left step and the other half of the cycle taking a wider right-to-left step, resulting in an overall average that stays fairly constant. It wasn't until this metric was plotted relative to base position and velocity that the dependence on base position became evident. 
Some participants changed strategies. Recall that Figure 2 illustrated the FFT ratios for each participant, calculated over the last ten minutes of the trial. When the same calculation was performed in twenty-second increments across the entire twenty-minute trial, we observed that seven FTB walkers could have been classified as FIS walkers early in the trial. Some of these individuals switched strategies within a minute of motion onset. Others shifted gradually over the first half of the trial. There were no examples of a conversion in the other direction, from FTB to FIS.

Exploring innate differences in visual and proprioceptive sensory weighting in the normal population may allow us to tailor training by targeting the most effective sensory modality for each individual.

Adaptation styles are shared here to highlight the unique human response to a novel environment. Our data support those of Lambrey and Berthoz [15], which illustrated that "subjects used conflicting visual and non-visual information differently according to individual 'perceptive styles'”. These styles, or propensities, should be considered when designing similar adaptation studies to avoid inadvertently favoring one type of adapter over another. In rehabilitation, the reverse may be true. Perhaps rehabilitation efforts can be augmented by recognizing these innate locomotor preferences and tailoring individual therapy regimens accordingly.

A focus of our laboratory has been to facilitate the re-adaptation of astronauts to Earth’s gravity or the adaptation to a novel gravitational environment, such as the moon or another planetary surface. This re-adaptation process is often accompanied by varying degrees of gait ataxia [16-20]. We are exploring the use of gait adaptability training as a countermeasure to mitigate the effects of microgravity exposure on locomotion. Training with task variability and 
exposure to repeated sensorimotor challenges expedites adaptation through a motor learning process termed adaptive generalization [21]. Locomotor training via this mode of learning has been shown to enhance motor skills [22-24]. Similarly, we hope to train individuals to adapt more quickly to conditions that cannot be accurately simulated here on Earth by exposing them to different novel sensorimotor experiences while they walk. This has been explored successfully, on a more general level, in clinical rehabilitation. Hemiparetic stoke patients who performed a task-orientated exercise program under conditions of vision and surface manipulations showed more improvement in their standing balance than a control group that performed a conventional task-oriented program [25]. Patients with balance control problems [26-28] and gait disturbances [29, 30] have also demonstrated improvement following adaptive generalization training. We believe analogous improvements might be made in the reduction of gait ataxia in returning astronauts if they are trained to make rapid adjustments to novel physical and sensory stimuli such as that produced by variations in visual flow and support surface stability.

\section{Acknowledgements}

This work was supported by the National Space Biomedical Research Institute through NASA NCC 9-58.

\section{References}

1. Bunday, K.L., et al., The effect of trial number on the emergence of the 'broken escalator' locomotor aftereffect. Exp Brain Res, 2006. 174(2): p. 270-8.

2. Jensen, L., T. Prokop, and V. Dietz, Adaptational effects during human split-belt walking: influence of afferent input. Exp Brain Res, 1998. 118(1): p. 126-30.

3. MacLellan, M.J. and A.E. Patla, Adaptations of walking pattern on a compliant surface to regulate dynamic stability. Exp Brain Res, 2006. 173(3): p. 521-30. 
4. MacLellan, M.J. and A.E. Patla, Stepping over an obstacle on a compliant travel surface reveals adaptive and maladaptive changes in locomotion patterns. Exp Brain Res, 2006. 173(3): p. 531-8.

5. Mulavara, A.P., et al., Exposure to a rotating virtual environment during treadmill locomotion causes adaptation in heading direction. Exp Brain Res, 2005. 166(2): p. 2109.

6. $\quad$ Nomura, Y., et al., Optic flow dominates visual scene polarity in causing adaptive modification of locomotor trajectory. Brain Res Cogn Brain Res, 2005. 25(3): p. 624-31.

7. Prokop, T., M. Schubert, and W. Berger, Visual influence on human locomotion. Modulation to changes in optic flow. Exp Brain Res, 1997. 114(1): p. 63-70.

8. $\quad$ Richards, J.T., Mulavara, A.P., Bloomberg, J.J., The interplay between strategic and adaptive control mechanisms in plastic rehabilitation of locomotor function. Exp Brain Res, 2007. 78(3): p. 326-38.

9. Thies, S.B., J.A. Ashton-Miller, and J.K. Richardson, What causes a crossover step when walking on uneven ground? A study in healthy young women. Gait Posture, 2007. 26(1): p. 156-60.

10. Warren, W.H., B.A. Kay, and E.H. Yilmaz, Visual control of posture during walking: functional specificity. J Exp Psychol Hum Percept Perform, 1996. 22(4): p. 818-38.

11. Dijkstra, T.M., et al., Frequency dependence of the action-perception cycle for postural control in a moving visual environment: relative phase dynamics. Biol Cybern, 1994. 71(6): p. 489-501.

12. Keshner, E.A., R.V. Kenyon, and J. Langston, Postural responses exhibit multisensory dependencies with discordant visual and support surface motion. J Vestib Res, 2004. 14(4): p. 307-19.

13. Masterton, B.A. and G.B. Biederman, Proprioceptive versus visual control in autistic children. J Autism Dev Disord, 1983. 13(2): p. 141-52.

14. van Hedel, H.J. and V. Dietz, The influence of age on learning a locomotor task. Clin Neurophysiol, 2004. 115(9): p. 2134-43.

15. Lambrey, S. and A. Berthoz, Combination of conflicting visual and non-visual information for estimating actively performed body turns in virtual reality. Int $\mathrm{J}$ Psychophysiol, 2003. 50(1-2): p. 101-15.

16. Bloomberg, J.J. and A.P. Mulavara, Changes in walking strategies after spaceflight. IEEE Eng Med Biol Mag, 2003. 22(2): p. 58-62.

17. Chekirda, I.F. and A.V. Eremin, [Dynamics of cyclic and acyclic locomotion by the crew of "Soyuz-18" after a 63-day space flight]. Kosm Biol Aviakosm Med, 1977. 11(4): p. 913.

18. Kozlovskaya, I.B., et al., Pathophysiology of motor functions in prolonged manned space flights. Acta Astronaut, 1981. 8(9-10): p. 1059-72.

19. Layne, C.S., P.V. McDonald, and J.J. Bloomberg, Neuromuscular activation patterns during treadmill walking after space flight. Exp Brain Res, 1997. 113(1): p. 104-16.

20. Reschke, M.F., et al., Space flight and neurovestibular adaptation. J Clin Pharmacol, 1994. 34(6): p. 609-17.

21. Welch RB, B.B., Anand S, Browman KE Alternating prism exposure causes dual adaptation and generalization to a novel displacement. Percept Psychophys, 1993. 54: p. 195-204. 
22. Cohen, H.S., J.J. Bloomberg, and A.P. Mulavara, Obstacle avoidance in novel visual environments improved by variable practice training. Percept Mot Skills, 2005. 101(3): p. 853-61.

23. Lam T, D.V., Transfer of motor performance in an obstacle avoidance task to different walking conditions. J Neurophysiol, 2004. 92: p. 2010-2016.

24. van Hedel HJA, B.M., Erni T, Dietz V Obstacle avoidance during human walking: transfer of motor skill from one leg to the other. J. Physiol, 2002. 543: p. 709-717.

25. Bayouk, J.F., J.P. Boucher, and A. Leroux, Balance training following stroke: effects of task-oriented exercises with and without altered sensory input. Int J Rehabil Res, 2006. 29(1): p. 51-9.

26. Silsupadol, P., et al., Training of balance under single- and dual-task conditions in older adults with balance impairment. Phys Ther, 2006. 86(2): p. 269-81.

27. Suarez, H., A. Suarez, and L. Lavinsky, Postural adaptation in elderly patients with instability and risk of falling after balance training using a virtual-reality system. Int Tinnitus J, 2006. 12(1): p. 41-4.

28. Pavlou M, L.A., Davies RA, Gresty MA, Bronstein AM. , Simulator based rehabilitation in refractory dizziness. J Neurology, 2004. 251: p. 983-995.

29. Baram, Y. and A. Miller, Virtual reality cues for improvement of gait in patients with multiple sclerosis. Neurology, 2006. 66(2): p. 178-81.

30. Fung, J., et al., A treadmill and motion coupled virtual reality system for gait training post-stroke. Cyberpsychol Behav, 2006. 9(2): p. 157-62. 


\section{Captions for Figures}

Figure 1. A) Lateral torso position superimposed on base position for representative FTB and FIS walkers. B) When base position is subtracted from torso position, a characteristic waveform for each group emerges. C) FFT of the adjusted torso position shown in B for the same subjects (lower frequency). The high frequency represents stride frequency.

Figure 2. Amplitude ratios at base frequency for the last ten minutes of each subjects' trial. FTB walkers have lower ratios $(<0.04)$ while FIS walkers have higher ratios $(>0.04)$.

Figure 3. Polar plots of right-foot-to-left-foot step width for the same FTB and FIS walkers. The amplitude of the individual data points and the radii of the fit circles are normalized to each subject's step width obtained during the pre-walk period. Right-foot-to-left-foot step width from the pre-walk period (baseline step width) is represented by the unity circle, shown in gray.

Figure 4. Dots indicate the centers of the best-geometric-fit circles representing the final ten minutes of data for each participant. Shaded areas indicate \pm 1 SD around the mean of the angular position of the data points for the FIS and FTB groups. FIS walkers show a downward shift in their best-geometric-fit circles, indicating that the left foot steps wider when the base is moving right and narrower when the base is moving left. Step width for FTB walkers is either not affected signficantly by the base motion or has the opposite tendency of the FIS walkers. 

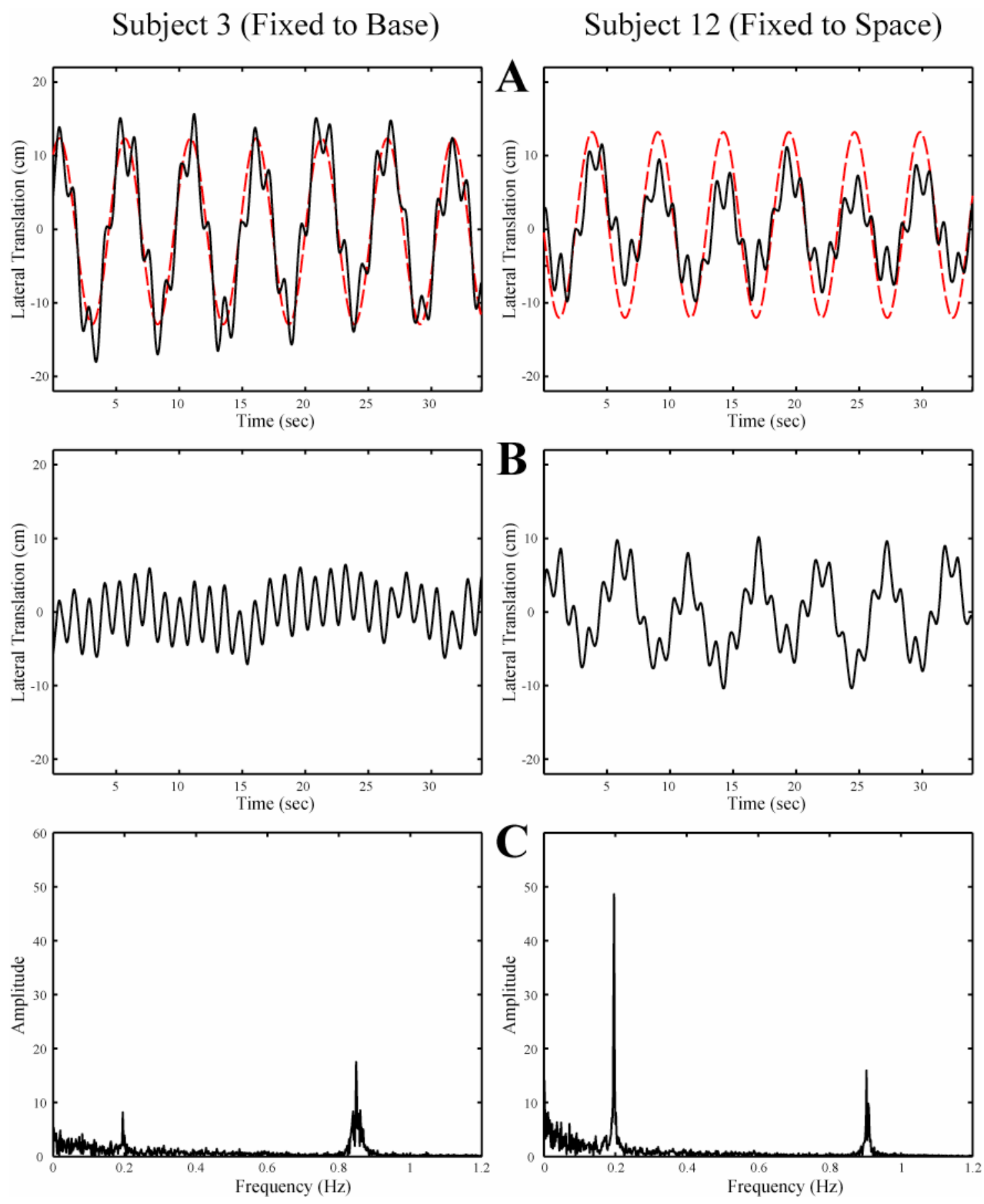


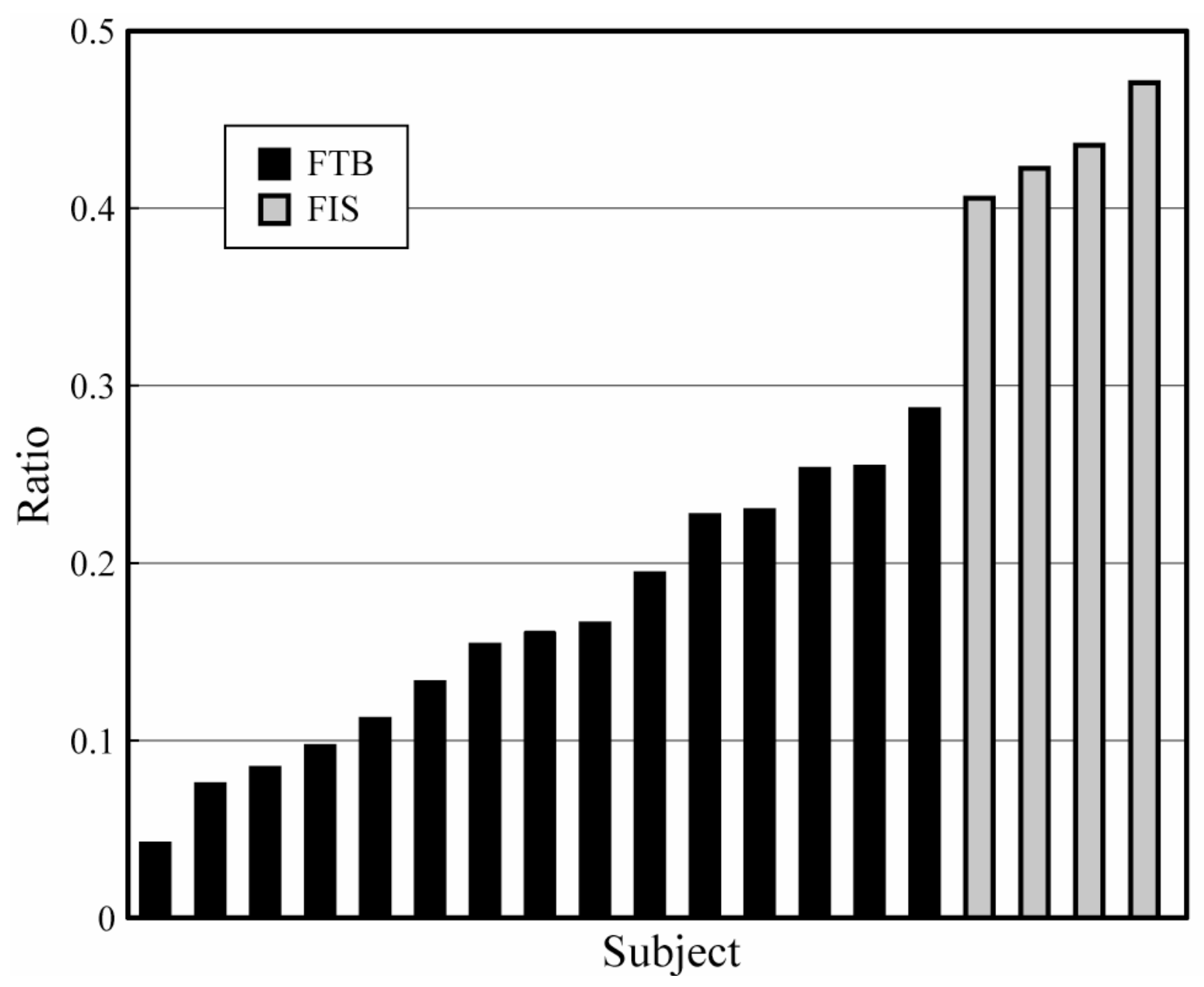



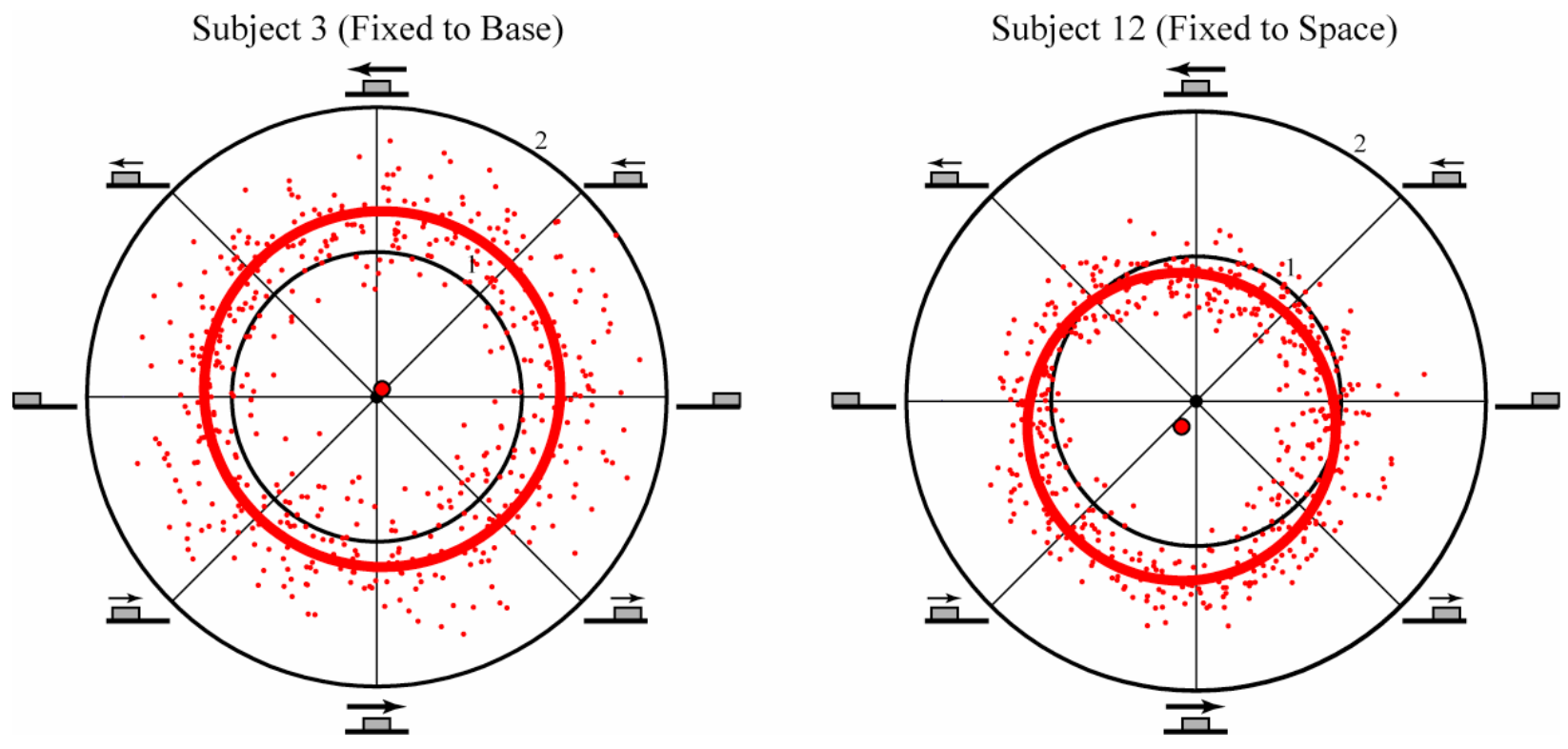


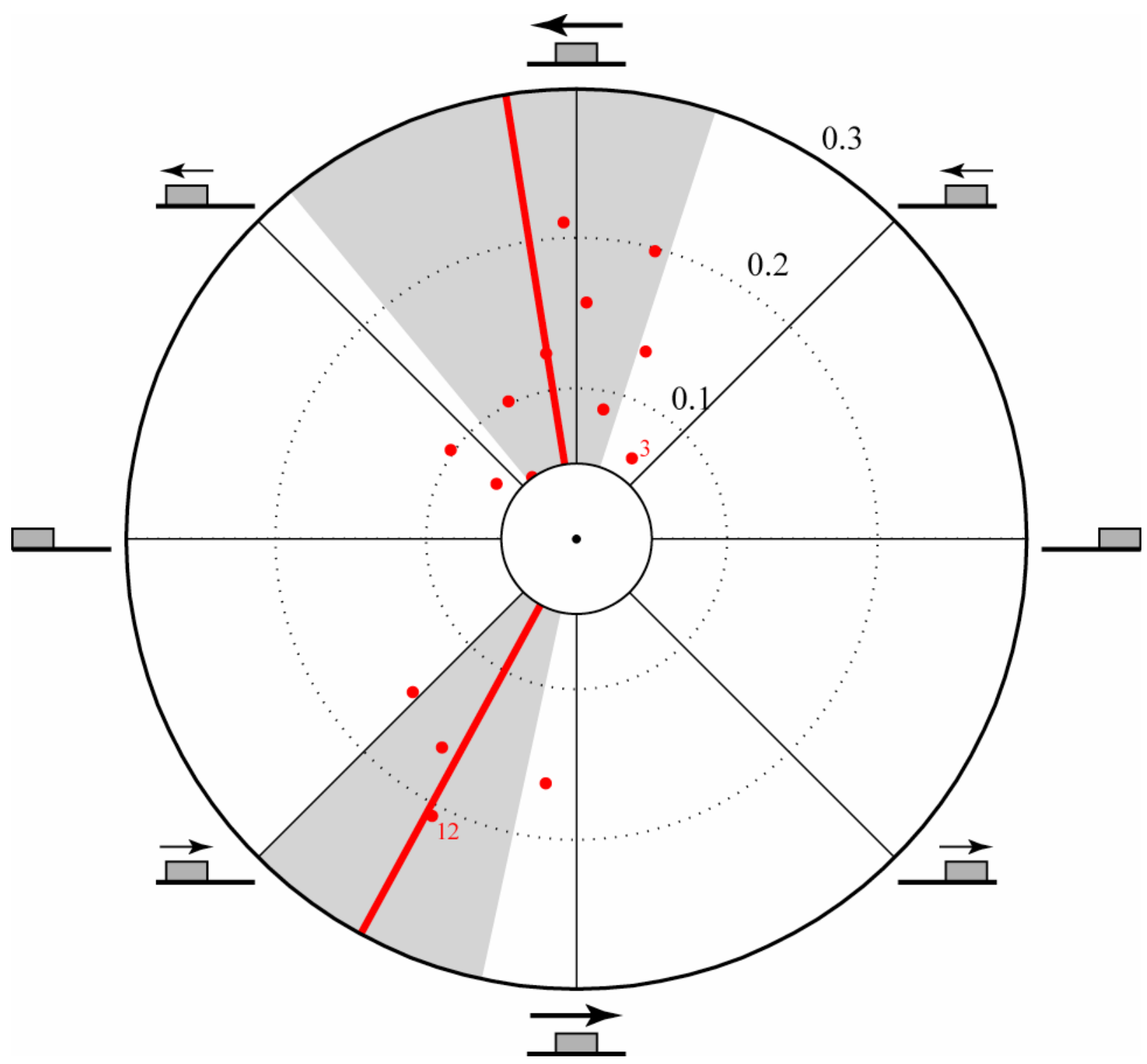

